\title{
Modeling and Simulation of the Communication Channel of
}

\section{Spacecraft Reentry}

\author{
Honghui $\mathrm{Mu}$ \\ Changchun University of science and technology, Changchun 130000, China \\ 270331219@qq.com
}

\begin{abstract}
This paper introduces the reasons and classification of wireless space fading, statistical characteristics of different types of channels. Through the analysis of reentry channel characteristics, we select the two pathes frequency selective slow fading channels.The spectral density function of Doppler power about two scattering path is derived.Channel model is established by rician method.Then determine the channel model parameters and simulate the wireless fading channel model with Matlab / Simulink software
\end{abstract}

Keywords:Reentry process,wireless fading channel,rician method

\section{1. the introduction}

Since 1999,when Shenzhou spacecraft was successfully launched, China has opened the prelude of deep space exploration. with the development of the aviation industry, When spacecraft return, which passed four stages: aberrancy, interim ,reentry and landing . When entering the reentry vehicle, the communication between Aircraft and ground control station is special. Firstly, The electromagnetic wave spread in wireless channel, which occur Reflection, Scattering and Diffraction with the ground, mountains, trees and other obstacles reflection. The ground stations received a superposition of multiple paths, which called multipath effect. Because the distance between the aircraft and station so far, make the time delay between different path is bigger. Secondly, the capsule reentry return, it has the characteristics of high speed and dynamic change, so there is a high dynamic Doppler effect. Thus it can be seen that study the anti fading channel is the key to the stable and reliable landing of spacecraft.

\section{2. wireless spatial channel characteristics}

When the spacecraft into reentry period, it communicate with the ground control station that is wireless channel. Due to the influence of multipath effect, different path components that amplitude, phase and arrival time and the incidence angle are different. They are make the received composite signal in amplitude and phase have a serious distortion, which occurred in the multipath fading.

\section{1 wireless Spatial Channel Characteristics}

Multipath effect can generate the time dispersion effect and frequency selective fading, Doppler effect will generate the frequency dispersion effect and the time 
selective fading [2], which are called the small scale fading. The curve which amplitude change fast and larger is the small scale fading curve, according to the characteristics of the spacecraft in the reentry communication, this paper mainly introduces the small scale fading.

According to the relationship between the coherence bandwidth $B_{c}$ and signal bandwidth of the symbol $B_{m}$ [1], the multipath fading channel can be divided into two categories:

(1) $B_{m} \ll B_{c}$, the channel is called flat fading channel. In this case, the delay between different paths is very small.

(2) $B_{m} \geq B_{c}$, the channel is called the frequency selective fading channel. In this case, the channel causes serious distortion of the signal. The small scale fading and Large scale fading as shown Figure 1.

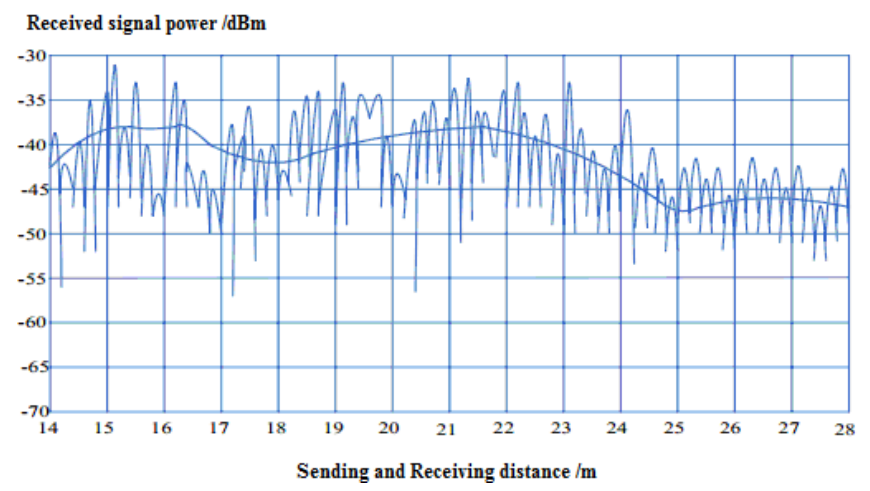

Figure 1 the small scale fading and large scale fading

\section{2 flat fading channel model}

The flat fading channel is complex Gauss random channel that composed of two real gauss random process, the PDF of the amplitude is the Rayleigh / rice distribution. So the flat fading channel can be modeled by the rice method that as shown in Figure 2, $\rho$ is Amplitude fading of direct path, $f_{\rho}$ is Doppler shift of direct path, $\theta_{\rho}$ is phase shift of direct path.

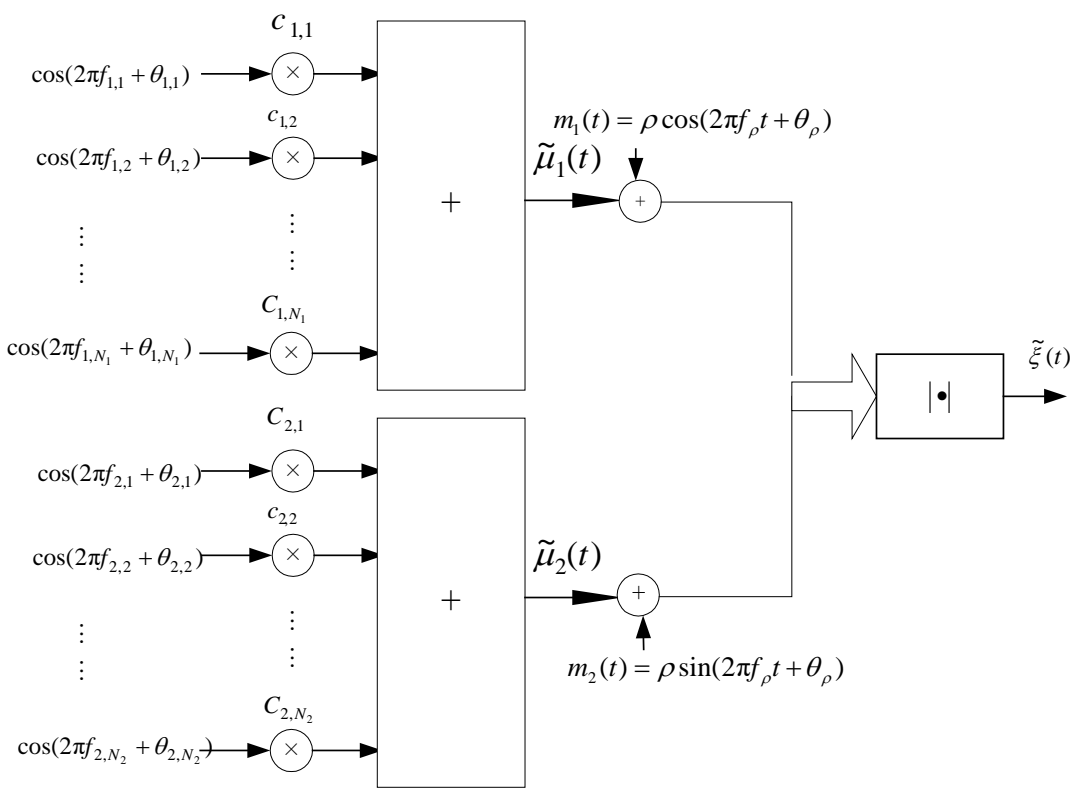

Figure 2 rayleigh/rice channel model 
Because the direct path $m_{i}(t)$ is a time variant stochastic process, considering the rice complex random variable

$$
\tilde{\mu}_{\rho}(t)=\tilde{\mu}_{\rho_{1}}(t)+j \tilde{\mu}_{\rho_{2}}(t)
$$

Therefore, the amplitude and phase probability density function as below:

$$
\begin{gathered}
\tilde{p}_{\xi}(z)=4 z \int_{-\pi}^{\pi}\left\{\int_{0}^{\infty} \prod_{n=1}^{N_{1}}\left[J_{0}\left(2 \pi c_{1, n} v_{1}\right)\right] g_{1}\left(z, \theta, v_{1}\right) d v_{1}\right\} \\
\cdot\left\{\int_{0}^{\infty} \prod_{m=1}^{N_{2}}\left[J_{0}\left(2 \pi c_{2, m} v_{2}\right)\right] g_{2}\left(z, \theta, v_{2}\right) d v_{2}\right\} d \theta \\
\tilde{p}_{\theta}(\theta)=4 \int_{0}^{\infty}\left\{z \int_{0}^{\infty} \prod_{n=1}^{N_{1}}\left[J_{0}\left(2 \pi c_{1, n} v_{1}\right)\right] g_{1}\left(z, \theta, v_{1}\right) d v_{1}\right\} \\
\cdot\left\{\int_{0}^{\infty} \prod_{m=1}^{N_{2}}\left[J_{0}\left(2 \pi c_{2, m} v_{2}\right)\right] g_{2}\left(z, \theta, v_{2}\right) d v_{2}\right\} d z
\end{gathered}
$$

When $C_{i, n}=\sigma_{i} \sqrt{2 / N_{i}}, \quad \sigma_{i}^{2}=1, f_{\rho}=0, N_{1}=7, N_{2}=8, \quad \theta_{\rho}$ is random number which range is $[0,2 \pi)$.Through the simulation of different $\rho$,the probability density distribution and Autocorrelation function are obtained, as shown in Figure 3.
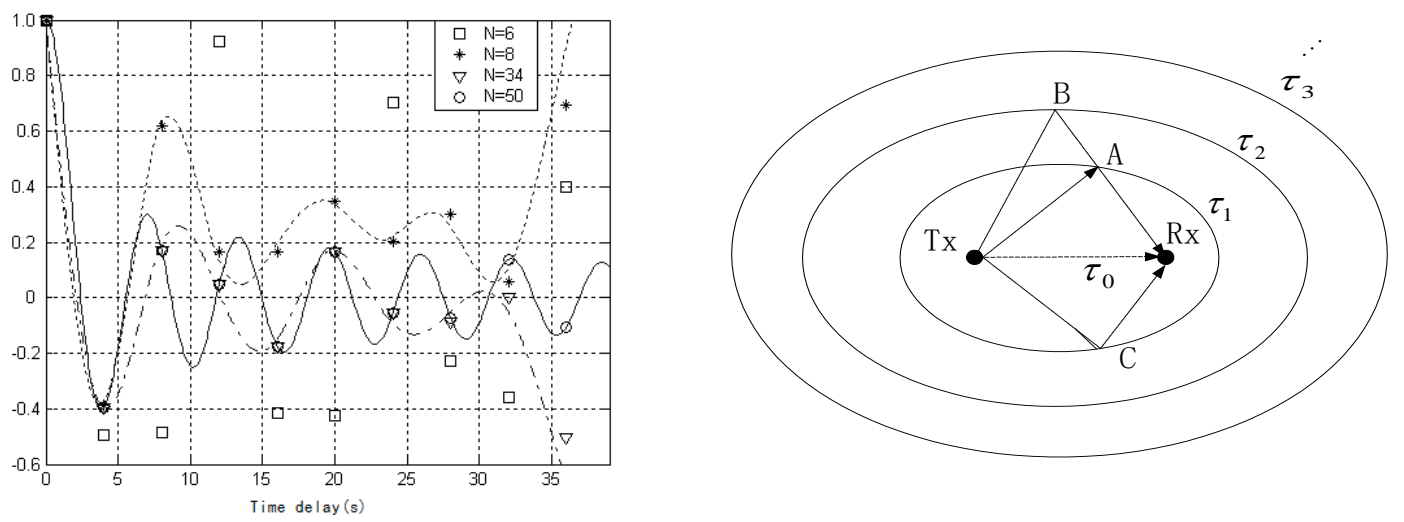

Figure 3 the PDF and autocorrelation function Figure 4 elliptical scattering model

\section{3. $\mathbf{3}$ frequency selective fading channel model}

Parsons and Bajwa proposed an elliptical scattering model, as shown in Figure 4. When path a lot, have a lot of numbers of delay $\tau_{n}(t)$.

the focus, and all of the ellipses are common focus, and the circumference of each ellipse is a collection of all the same points in the distance to the focal point. According to the time variant impulse response of the elliptic model and the frequency selective fading channel, the channel model can be described as:

$$
h(\tau, t)=\sum_{l=0}^{L-1} \beta\left(\tau_{l}\right) c_{l}(t) \delta\left(\tau-\tau_{l}\right)
$$

$c_{l}(t)$ indicates the path component of the delay for $\tau_{l}$, which can be expressed as follows:

$$
c_{l}(t)=\sum_{n=1}^{N_{l}} b_{n, l} e^{j\left(2 \pi f_{n, l} t+\theta_{n, l}\right)}
$$

$b_{n, l} 、 f_{n, l} 、 \theta_{n, l}$ respectively represents the amplitude attenuation, the Doppler shift and the phase shift for the $\mathrm{N}$ path in the $\tau_{l}$ path, the $c_{l}(t)$ can be modeled as a flat 
fading channel model. $h(\tau, t)$ can be modeled as a discrete tapped delay line model, as shown in Figure 5.

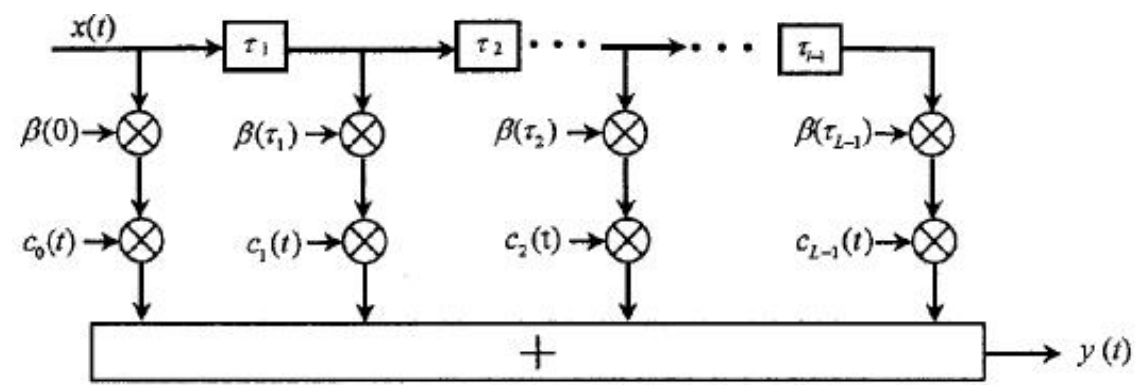

Figure 5 frequency selective channel model

\section{4. modeling and simulation of the downlink communication channel in reentry phase}

The multipath phenomenon of the downlink communication link in the return module as shown in Figure 6. Due to the altitude of receiving antenna as low, the delay between the path and the direct path is very small, so it can be ignored. But the delay between the mountains reflected path and direct path is longer, that will generate code asked crosstalk. Therefore, the downlink channel should be modeled as a frequency selective slow fading channel in the reentry process. Through the analysis, return cabin of downlink can be modeled as two channel models, the first the first path is zero delay path, mainly produced by direct path and the antenna near a ground reflection and scattering. The second path for the large time delay, mainly produced by mountains, clouds and other large obstacles reflection and diffraction.

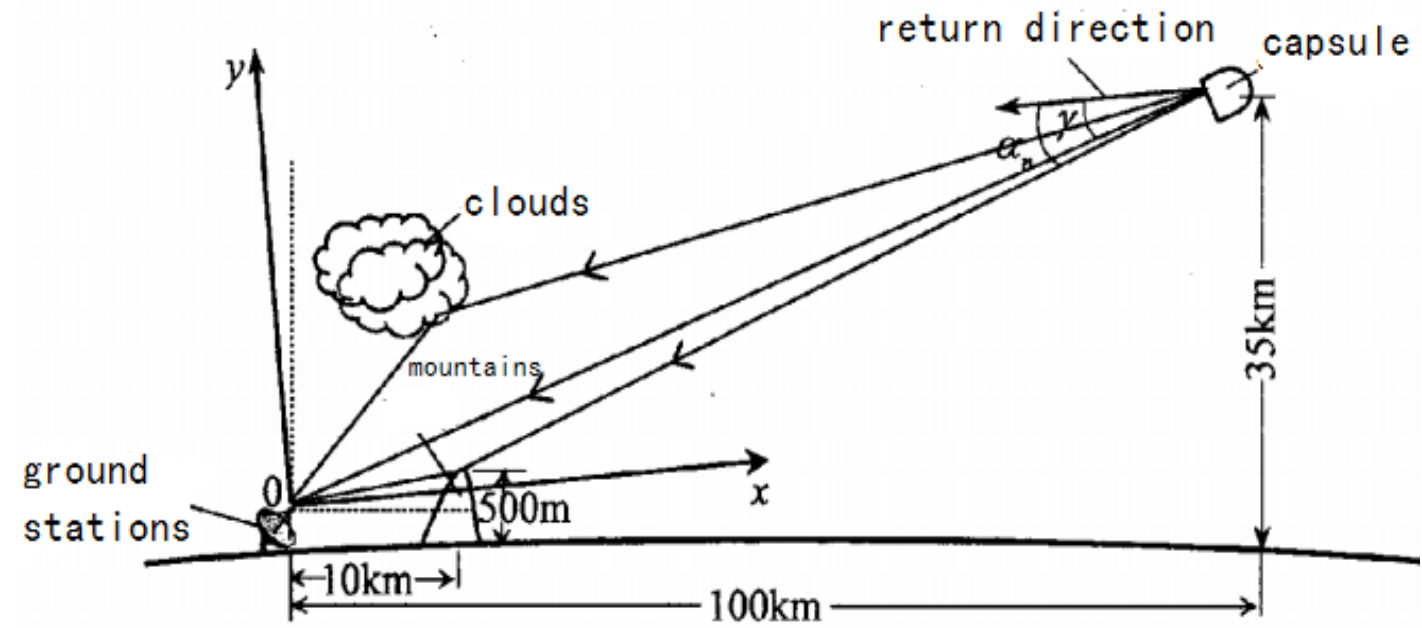

Figure 6 the multipath phenomenon of capsule downward communication link channel

From the above analysis, the key of two paths fading channel modeling is how to determine the delay coefficient $\beta\left(\tau_{l}\right)$ and the delay path $C_{l}(t)$ so that the path 
model is more close to the actual channel characteristics. $C_{l}(t)$ can be expressed as

$$
C_{l}(t)=\sum_{n=1}^{N_{l, 1}} a_{n, l, 1} \cos \left(2 \pi f_{n, l, 1} t+\theta_{n, l, 1}\right)+j \sum_{n=1}^{N_{l, 2}} a_{n, l, 2} \cos \left(2 \pi f_{n, l, 2} t+\theta_{n, l, 2}\right)
$$

Equal area method be used to Calculate the Doppler frequency coefficient $f_{n, l, i}$, Doppler amplitude coefficient $a_{n, l, i}$, Doppler phase shift calculation $\theta_{n, l, i}$ :

$$
f_{n, l, i}=\left\{\begin{array}{l}
f_{\max } \sin \left(\frac{\pi n}{2 N_{l, i}}\right) \quad \mathrm{l}=0, n=1,2, \ldots, N_{i} \\
f_{\max } \sin \left(\frac{\pi n}{2 N_{l, i}}+\frac{\pi}{36}\right) \quad \mathrm{l}=1,0<n \leq\left[\frac{8}{9} \cdot N_{l, i}\right] \\
f_{\max } \sin \left(\frac{\pi\left(n-\left[\frac{8}{9} \cdot N_{l, i}\right]\right.}{4 N_{l, i}}+\frac{17 \pi}{36}\right) \quad \mathrm{l}=1,\left[\frac{8}{9} \cdot \mathrm{N}_{l, i}\right]<n \leq N_{l, i}
\end{array}\right.
$$

Where $\left[\frac{8}{9} . N_{l, i}\right]$ represents the integer down. Because the value of $\theta_{n, l, i}$ does not affect the power spectral density, it takes a uniform distribution of $(0,2 \pi)$ on the random number. Finally, the delay coefficient $\beta\left(\tau_{l}\right)$ can be determined by the time delay power spectral density function.

Simulate the downlink communication channel of the reentry phase by Simulink .There are two paths, the first path is the zero time delay path including the direct path, the Doppler frequency is $f_{\rho}=f_{\max } \cos (\gamma)$, the amplitude attenuation is $\rho$, the amplitude probability density obeys the Rican distribution. The amplitude probability density function obeys the Rayleigh distribution for the second path and the delay time is $\tau_{1}$. The model is as follows:

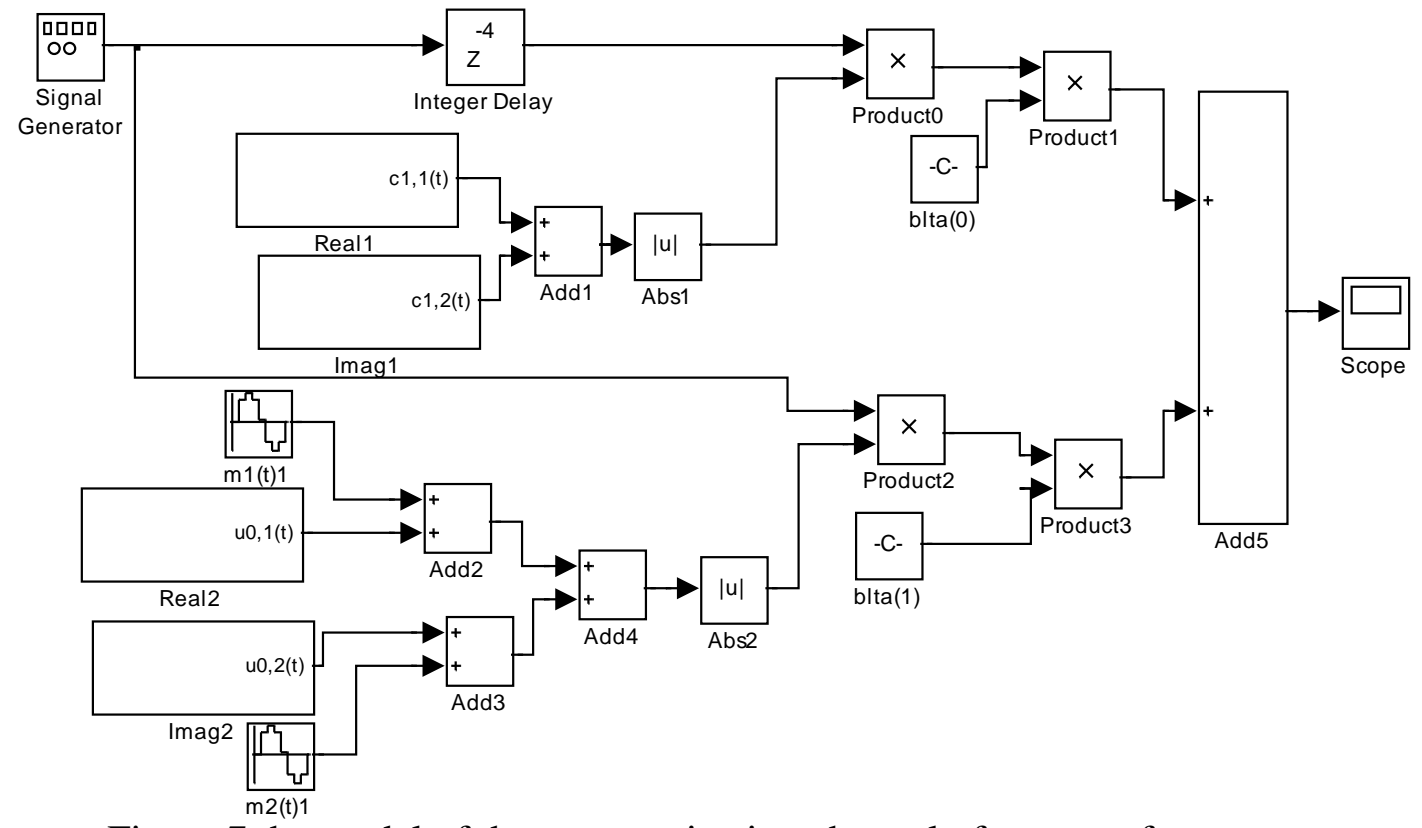

Figure 7 the model of the communication channel of spacecraft reentry 
In above picture,Doppler frequency coefficient $a_{n, l, i}$, Doppler frequency coefficient $f_{n, l, i}$, Delay time $\beta\left(\tau_{l}\right)$ can respectively through formula (7), formula (8) and formula (9). The real and imaginary parts of the Rician/Rayleigh channel are generated by two random processes which obey Gauss distribution, there, the real parts of Rician $N_{0,1}=7$, the imaginary parts of Rician $N_{0,2}=8$.the real parts of Rayleigh $N_{1,1}=9$, the imaginary parts of Rayleigh $N_{1,2}=10$.

\section{4、 system simulation analysis}

Simulated the channel model when verified at the altitude of $H=35 \mathrm{~km}$. At this time, the maximum Doppler frequency shift is $15 \mathrm{KHz}$. The angle between the direct path and the direction of entry $\gamma=30^{\circ}$, the amplitude attenuation $\rho=0 \mathrm{~dB}$, the zero time delay $K_{0}=5 d B$, when the time delay $\tau_{1}=2 \mu \mathrm{s}$, then $K_{1}=25 d B$. In addition, the simulation time is $5 \mathrm{~ms}$, and the sampling time is $0.5 \mu \mathrm{s}$. As shown in Figure 8 and Figure 9.
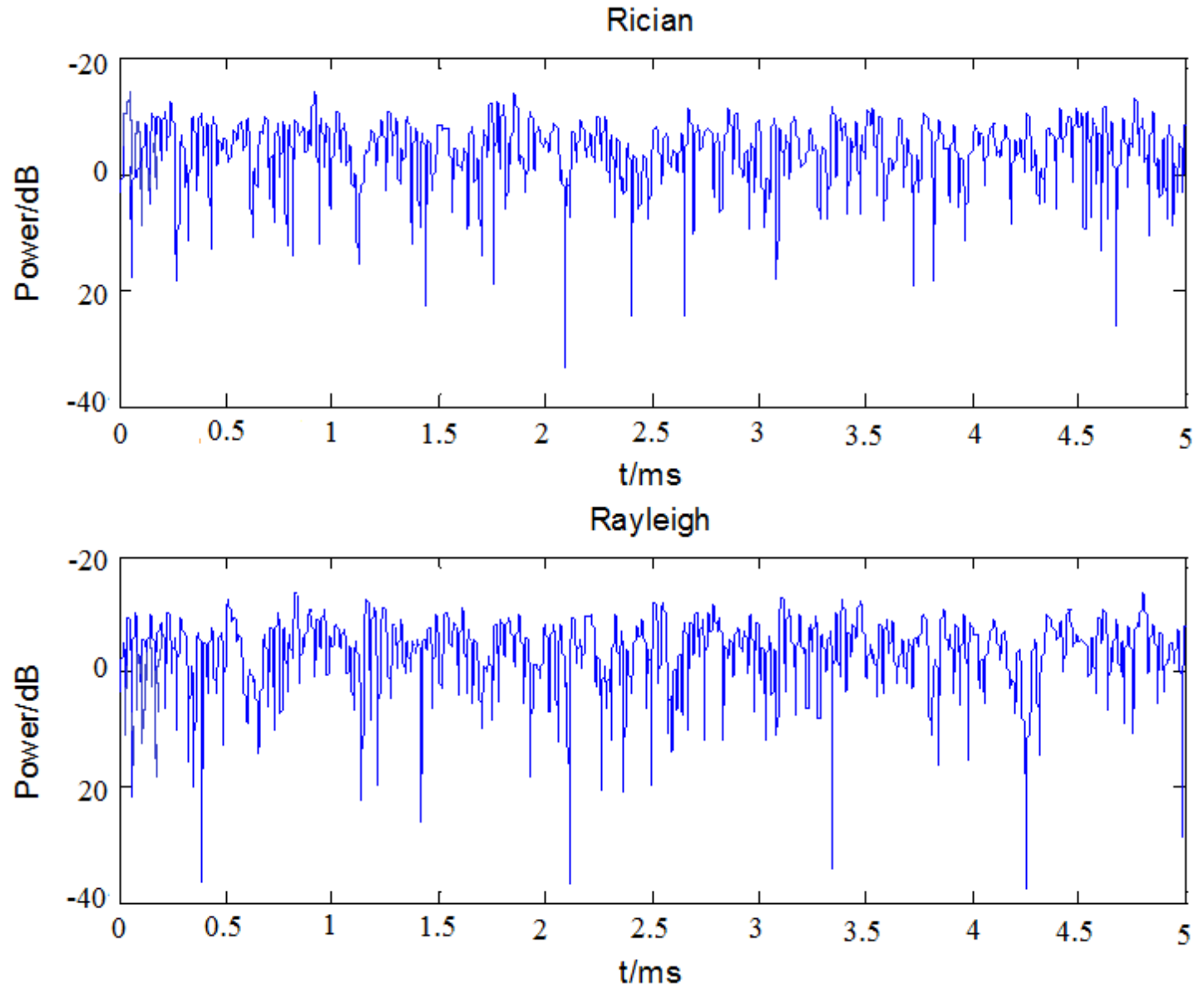

Figure 8 simulation of fuzzy self-turning PID control curve 


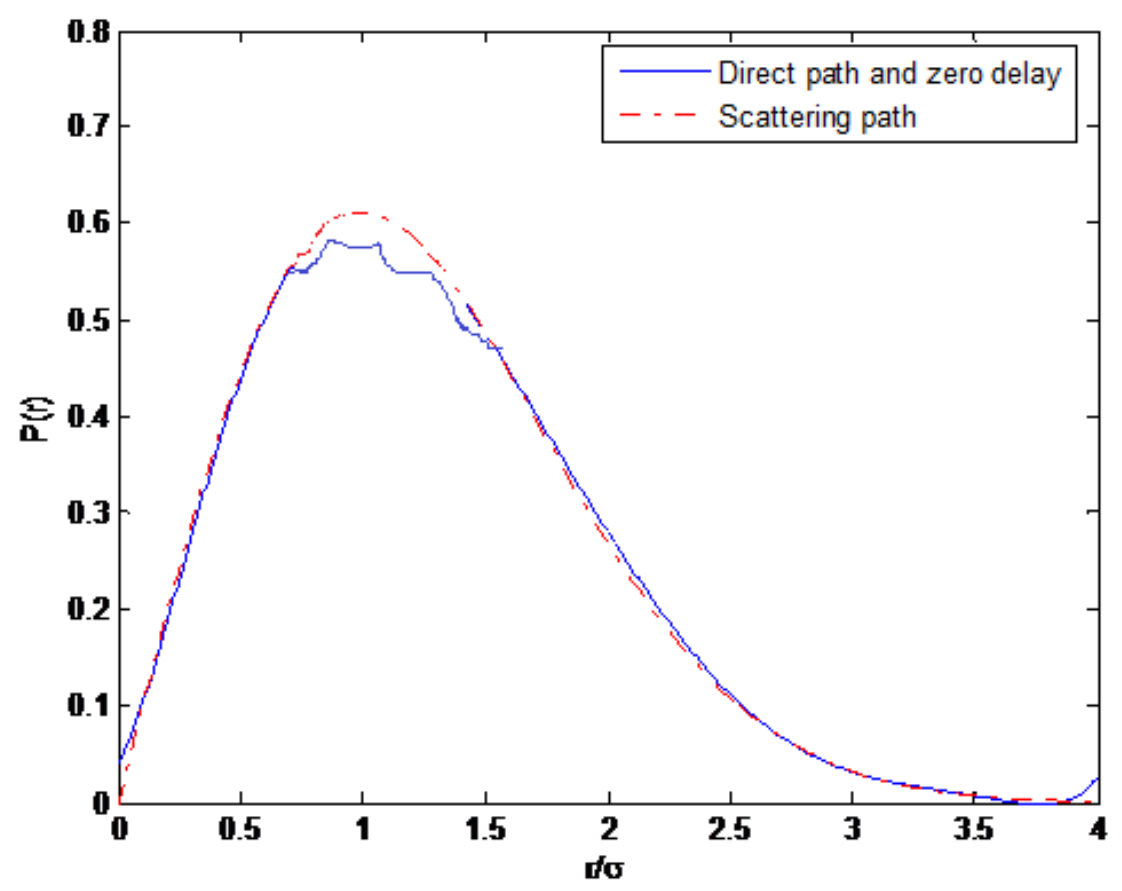

Figure 9 the PDF of the two paths

\section{4、 conclusion}

In this paper, established the Gauss channel model,Flat fading channel model and Frequency selective channel model.The statistical properties of each model are derived. Then, the wireless space in the downlink communication channel is modeled as a wireless space. Two path frequency selective slow fading channel, determine the Doppler frequency and the amplitude and the phase delay line coefficient and channel model,The simulation and test of the channel model are completed in Simulink / Matlab.

\section{5、 Reference}

[1] Ling Zheng, Qing Zhao.Simulation of the Electromagnetic Waves Transmission in Plasma[J].International Conferenc onElectronic\&Mechanical Engineering and Information Technology,2011.

[2] Liu Tingting, Liu Shunlan. The Simulation Model of Ground-to-air Channel and Its Application in SC-FDE[J]. 2009 Second ISECS International Colloquium on Computing, Communication, Control, and Management (CCCM 2009).

[3] Patel,C.S,Stuber,G.L,Pratt,T.G. Simulation of Rayleigh-faded mobile-to-mobile communication channels[J]. Communications,IEEE Transactions on . 2005.

[4] Ying Guofeng. Research and design of communication channel simulator for spacecraft reentry [D]. Nanjing University of Science and Technology. 2014.

[5] Chengshan Xiao, "Second-Order Statistical Properties of the WSS Jakes' Fading Channel Simulator”,IEEE TRANSACTIONS ON COMMUNICATIONS, VOL. 50, NO. 6, JUNE 2002. 